\title{
Evaluation of myocardial, hepatic, and renal perfusion in a variety of clinical conditions using an intravenous ultrasound contrast agent (Optison) and second harmonic imaging
}

\author{
J Hancock, H Dittrich, D E Jewitt, M J Monaghan
}

\begin{abstract}
Objective-To assess the potential of intravenous Optison, a second generation ultrasound contrast agent, and various ultrasound imaging modes to determine myocardial, kidney, and liver perfusion in normal subjects and patients with left ventricular dysfunction or chronic pulmonary disease together with renal or hepatic dysfunction.

Methods-Five normal subjects and 20 patients underwent grey scale echocardiographic imaging of myocardium, kidney, and liver during 505 intravenous injections of Optison. Images were assessed qualitatively by two independent observers and quantitatively using video densitometry to determine the peak contrast enhancement effect.
\end{abstract}

Results-Qualitative analysis showed that intermittent harmonic imaging was superior to either conventional fundamental or continuous harmonic imaging for all organs. Quantitative analysis showed that the peak change in echocardiographic intensity $v$ baseline during continuous harmonic imaging was 11 units for myocardium ( $p<0.03), 7$ units for kidney (NS), and 14 units for liver $(p<0.05)$. During intermittent harmonic imaging the peak change was significantly greater, being 33 units for myocardium $(\mathrm{p}<0.0001), \quad 24$ units for kidney $(p<0.0002)$, and 16 units for liver $(\mathrm{p}<0.001)$.

Conclusions-Organ tissue perfusion can be demonstrated following intravenous injection of Optison, particularly when used in combination with intermittent harmonic imaging techniques. This contrast agent is effective in a variety of clinical conditions.

(Heart 1999;81:636-641)

Keywords: ultrasound; contrast enhancement; echocardiography; Optison

To date, myocardial contrast echocardiography has had limited clinical applications because of the need to inject the contrast agent directly into the aorta or coronary arteries. More recently, the development of new ultrasound contrast agents with microsphere bubbles which are stable in vivo and which can retain their backscatter properties during transpul- monary transit has made endocardial definition and myocardial opacification following intravenous injection possible. ${ }^{1}$

In addition there have been advances in ultrasound technology. First, the discovery that, at diagnostic ultrasound intensities, contrast microbubbles resonate and create backscatter signals, not only at the fundamental (transmitted) frequency but at harmonics of that frequency. ${ }^{2}$ Although the backscatter is less at the harmonic frequencies than at the fundamental frequency, because tissue produces a smaller harmonic signal than contrast microspheres, the difference between tissue alone and tissue plus contrast agent is enhanced. Thus the development of imaging software and ultrasound transducers which transmit at the fundamental frequency and receive at the second harmonic frequency (twice the fundamental frequency) has improved the detection of intravenously injected contrast agents within the myocardium. ${ }^{3}$ Second, it has been observed that diagnostic ultrasound intensities may destroy contrast microspheres so that, by reducing the transmitting ultrasound intensity, the contrast effect is enhanced. ${ }^{4}$ This can be achieved by imaging intermittently using only one ultrasound frame for every one to five cardiac cycles. This allows destroyed contrast agent to be replenished in the tissue between frames and enhances the contrast effect.

The combination of the new transpulmonary contrast agents and the new ultrasound imaging techniques may make it possible to evaluate organ perfusion in humans following intravenous injections.

The aim of this study was to assess the efficacy of the contrast agent Optison (Molecular Biosystems Inc, San Diego, USA) in human subjects for myocardial, liver, and kidney perfusion following intravenous injection. Several animal studies of this agent have been reported, ${ }^{156}$ as well as initial safety studies in humans. ${ }^{7} 8$ Studies examining the role of this agent in patients have focused mainly on myocardial perfusion in the setting of coronary artery disease. ${ }^{9}$ Little is known about myocardial perfusion in patients with left ventricular dysfunction or chronic pulmonary disease, which may affect the transpulmonary passage of the contrast agent. The perfusion of other organs such as liver or kidney following intravenous injections of agents such as Optison has not been studied in detail in the human, particularly in the setting of hepatic or renal
Dr Hancock.

Accepted for publication 19 November 1998 
Table 1 Left ventricular ejection fraction, pulmonary artery systolic pressure, and hepatic and renal function in the patients studied

\begin{tabular}{|c|c|c|c|c|c|c|}
\hline Patient & $E F(\%)$ & $\begin{array}{l}P A S P(m m H g) \\
+\mathscr{F V P}\end{array}$ & $\begin{array}{l}\text { Bilirubin } \\
(\mu m o l / l)\end{array}$ & $\begin{array}{l}A S T \\
(I U / l)\end{array}$ & $\begin{array}{l}A L P \\
(I U / l)\end{array}$ & $\begin{array}{l}\text { Creatinine } \\
(\mu \mathrm{mol} / \mathrm{l})\end{array}$ \\
\hline 6 & $35-40$ & & & & & 86 \\
\hline 7 & $30-35$ & & & & & 100 \\
\hline 8 & $20-25$ & & & & & 100 \\
\hline 9 & $20-25$ & & & & & 105 \\
\hline 10 & $25-30$ & & & & & 101 \\
\hline 11 & $30-35$ & & & & & 120 \\
\hline 12 & $20-25$ & & & & & 114 \\
\hline 13 & $25-30$ & & & 302 & & 120 \\
\hline 14 & $20-25$ & & & & & 106 \\
\hline 15 & $25-30$ & & & 79 & 863 & 272 \\
\hline 16 & $45-50$ & 80 & 38.3 & & 192 & 115 \\
\hline 17 & $30-35$ & 64 & & & & 101 \\
\hline 18 & $25-30$ & 50 & & & & 111 \\
\hline 19 & $35-40$ & 50 & & 423 & 177 & 302 \\
\hline 20 & $25-30$ & 50 & & & & 313 \\
\hline 21 & $40-45$ & 52 & & & & 175 \\
\hline 22 & $35-40$ & 64 & & & & 239 \\
\hline 23 & $35-40$ & & & & & 544 \\
\hline 24 & $40-45$ & 50 & & & & 347 \\
\hline 25 & $30-35$ & & 44.6 & 114 & 145 & 151 \\
\hline
\end{tabular}

ALP, alkaline phosphatase (normal 30-120 IU/1); AST, aspartate aminotransferase (normal $<41$ $\mathrm{IU} / \mathrm{l}) ; \mathrm{EF}$, ejection fraction; PASP, pulmonary artery systolic pressure $(\mathrm{mm} \mathrm{Hg}+$ jugular venous pressure (JVP)).

dysfunction, though there are a few reports of animal studies with such agents. ${ }^{10-12}$ These agents also have the ability to delineate tumours within organs. ${ }^{13}$ Second harmonic imaging enhances these images. ${ }^{14}$ Optison can demonstrate liver and kidney perfusion with second harmonic imaging in animals, but its clinical efficacy has not been studied..$^{15}$ In this study we set out to address these points.

\section{Methods}

PATIENTS

Twenty five subjects were enrolled. Five were normal male volunteers (mean age 36 years, range 27 to 42 ). Twenty were patients (18 male, two female; mean age 58 years, range 32 to 78 ) with moderate to severe left ventricular dysfunction (ejection fraction $<40 \%$ ) or chronic pulmonary disease (including pulmonary hypertension with pulmonary artery systolic pressure $>40 \mathrm{~mm} \mathrm{Hg}$ ) or both. Ten

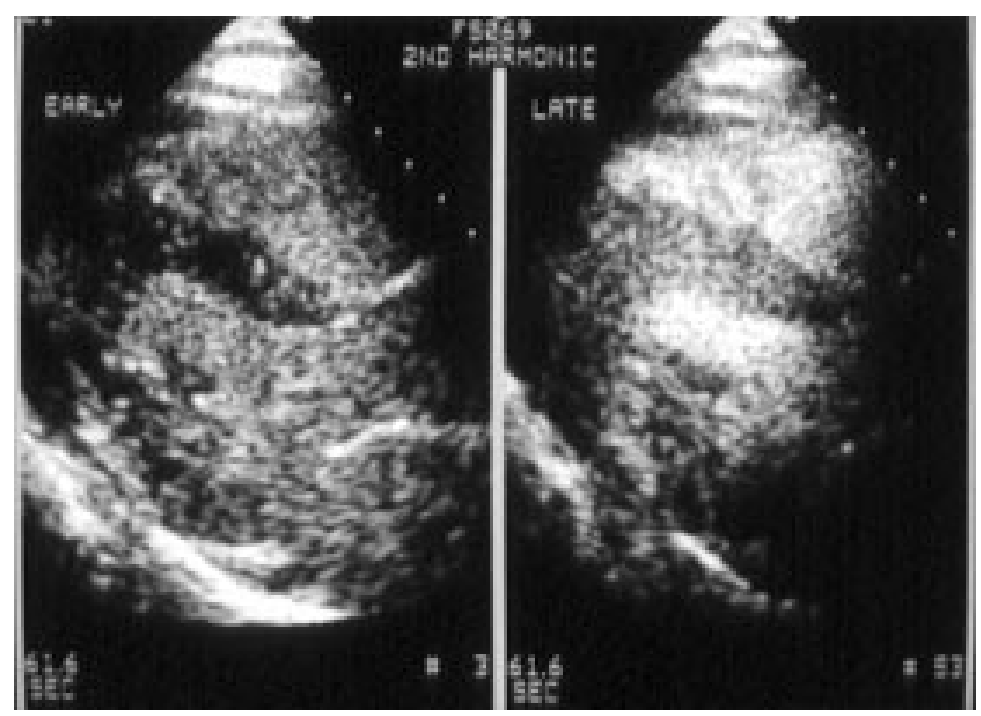

Figure 1 Myocardial opacification early (left) and late (right) after a $1 \mathrm{ml}$ injection of Optison during continuous harmonic imaging. Parasternal long axis and apical four chamber views are shown. Early after the injection of Optison, right and left ventricular cavities are opacified but no myocardial opacification is seen. Late after the injection, good myocardial opacification is demonstrated (score of 3 on visual analysis). had normal biochemical profiles and 10 had impaired renal function, impaired liver function on biochemical testing, or both (table 1). The project was approved by King's Healthcare ethics committee. All subjects gave written informed consent. Inclusion criteria included age 18 to 80 years and adequate baseline ultrasound images of heart, liver, and kidney. Exclusion criteria included pregnancy, lactation, known hypersensitivity to blood, blood products, or albumin, unstable angina, and acute myocardial infarction.

All subjects had a physical examination, a 12 lead ECG, blood biochemical and haematological analysis, and urinalysis before the study and then at 30 minutes and 24 hours after the study.

\section{CONTRAST AGENT}

Optison is a second generation contrast microsphere agent made by Molecular Biosystems Inc (MBI). It consists of octafluoropropane filled microspheres of mean diameter $2-5 \mu \mathrm{m}$ in a concentration of $1-10 \times 10^{8}$ particles $/ \mathrm{ml}$, prepared by the sonication of $1 \%$ human albumin in the presence of perfluoropropane (PFP). This agent has excellent backscatter properties and long in vivo persistence following intravenous injection. Clinical trials exemption was approved. Subsequently, Optison has been given a licence for sale in Europe and America.

The agent was kept suspended by continuous gentle agitation. Intravenous injections in a range of $0.1-5 \mathrm{ml}$ (total $45 \mathrm{ml}$ per patient) were given through an antecubital vein at a rate of less than $1 \mathrm{ml} / \mathrm{s}$, followed by a saline flush. Any adverse events during the study and up to 24 hours afterwards were noted. After the first eight patients it became apparent that it was unnecessary to use $45 \mathrm{ml}$ of contrast agent per patient in order to obtain satisfactory organ tissue enhancement, so the maximum dose was reduced to $20 \mathrm{ml}$ per patient. The dose was adjusted to achieve maximum contrast enhancement effect with minimal attenuation (optimal perfusion volume). Attenuation occurs when the concentration of contrast agent within the right or left ventricular cavity is so great that it creates an acoustic shadow behind it, obscuring all tissues which are further away from the ultrasound transducer. This effect almost always occurs at doses sufficient to produce contrast enhancement within the tissue but its persistence can be reduced by reducing the dose of contrast agent, such that attenuation occurs in the early phase following intravenous injection during peak opacification of the right and left ventricular cavities, but has receded by the time of peak contrast opacification of the tissue.

Overall, 505 intravenous injections of Optison were given (mean 20 per subject).

\section{ULTRASOUND IMAGING}

A prototype Hewlett Packard Sonos 2500 system (Hewlett Packard Inc, Andover, Massachusetts, USA) and an ATL HDI 3000 (ATL Inc, Bothell, Washington, USA) were used for grey scale ultrasonic imaging of myocardium, liver, and kidney. Images were recorded just 


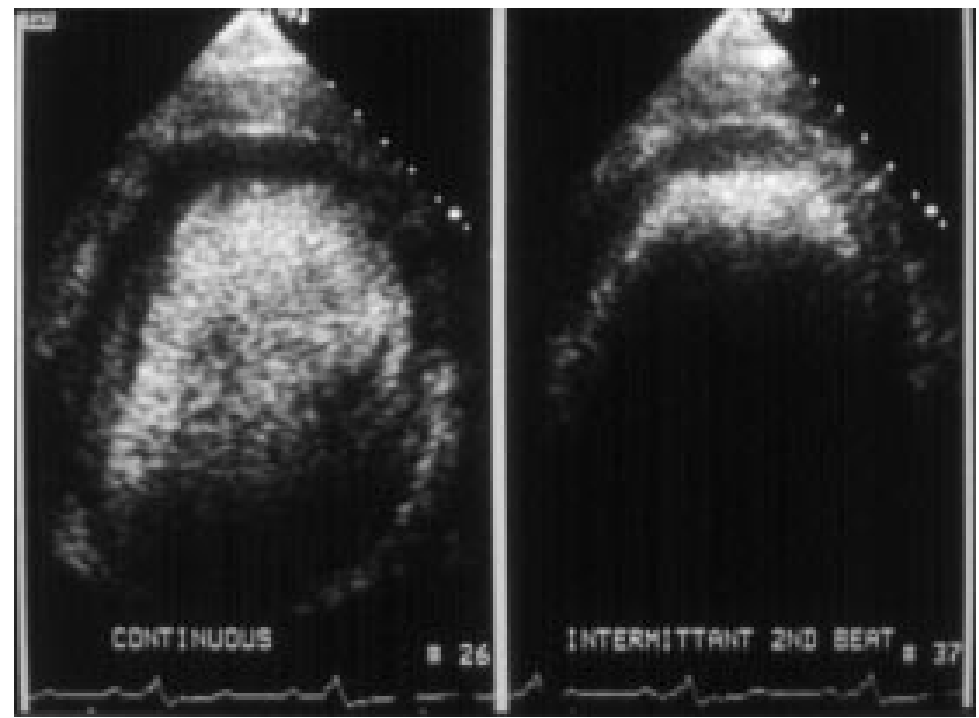

Figure 2 Myocardial opacification during continuous (left) and intermittent (right) harmonic imaging every second cardiac cycle following a $1 \mathrm{ml}$ injection of Optison. During continuous harmonic imaging, opacification of the left ventricular cavity, septum, and lateral wall is seen, but no opacification at the apex. During intermittent harmonic imaging, less contrast agent is destroyed, which results in visible perfusion at the apex and increased cavity attenuation.
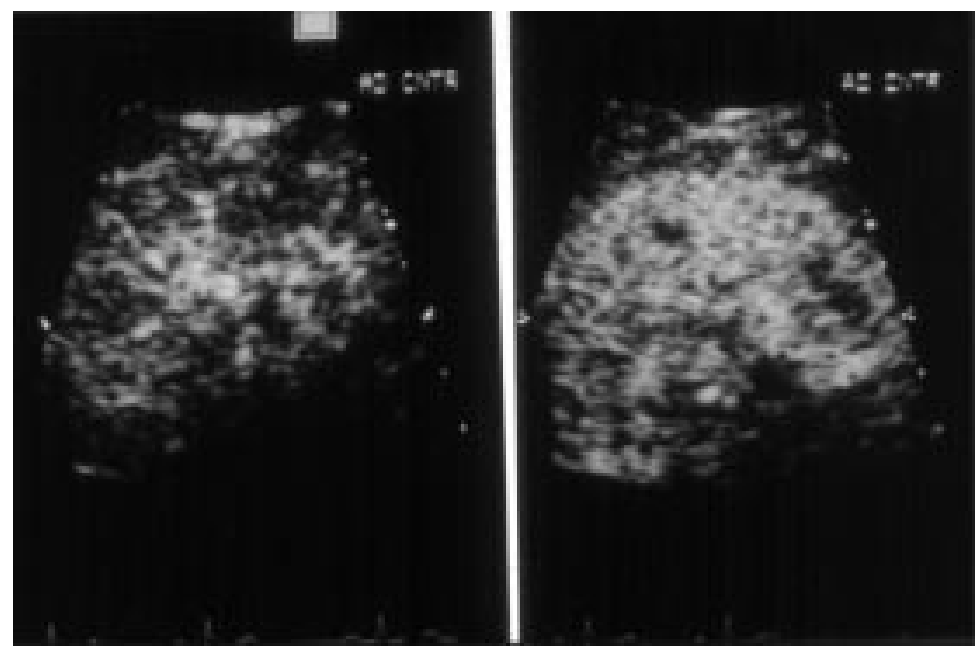

Figure 3 Renal opacification during continuous (left) and intermittent (right) harmonic imaging every second cardiac cycle following a $1 \mathrm{ml}$ injection of Optison. During intermittent imaging, less destruction of contrast agent results in increased tissue opacification
DATA ANALYSIS

Data were analysed qualitatively by two independent observers using a visual contrast enhancement scoring system where $0=$ no enhancement, 1 = faint enhancement, $2=$ moderate enhancement, $3=$ good enhancement, and 4 = attenuation, to determine the optimal perfusion volume for each organ in each subject. This was done in fundamental imaging mode. The peak contrast enhancement effect was scored using the same scale, once any effect of attenuation had subsided (that is, a score of 4 was not considered in assessing the peak contrast enhancement effect). This was done for all organs in all subjects at the optimal perfusion volume and using all imaging modes. Any disagreement between observers was resolved by consensus.

Video densitometry was performed using a Pentium PC workstation and Osiris medical imaging software, version 3.0, developed by the University Hospital of Geneva. This is available as shareware at internet site http:// expasy.hcuge.ch/UIN. A still video frame was "grabbed" and the ultrasonic grey scale level, representing echocardiographic intensity, measured within a region of interest drawn in a segment of the relevant tissue both before contrast injection and at peak contrast effect. This was repeated for all organs and all harmonic imaging modes for each subject. Organ perfusion was assessed by the change from baseline grey scale intensity.

STATISTICAL ANALYSIS

The pre and peak contrast effects for each organ in each imaging mode were compared using a paired $t$ test. Comparisons between imaging modes were performed using an unpaired $t$ test. A probability (p) value of $<0.05$ was taken to imply a significant difference.

\section{Results}

QUALITATIVE ANALYSIS

Visual analysis of the videotaped images was used to assess the optimal perfusion volume, deriving maximum opacification with minimal attenuation for each organ. This was $0.1-0.5$ $\mathrm{ml}$ for myocardium, $1 \mathrm{ml}$ for kidney, and 1-2 $\mathrm{ml}$ for liver in all patient groups.

With two observers using a visual scoring system to evaluate organ perfusion (fig 1), fundamental and continuous second harmonic imaging of all organs elicited faint opacification with contrast, with a score of 1.07 and 1.32 , respectively. In contrast, intermittent harmonic imaging provided the best visual evaluation of perfusion for all organs (figs 2 and 3), achieving a score of $2.23(\mathrm{p}<0.001)$ representing moderate to good (fig 4), though there was no significant difference between the intermittent imaging modes. For example the scores for continuous harmonic and intermittent harmonic imaging every one, two, or five beats for myocardium were $1.32,2.69,2.91$, and 3.33, respectively $(\mathrm{p}<0.0001$ for all intermittent modes $v$ continuous imaging). 


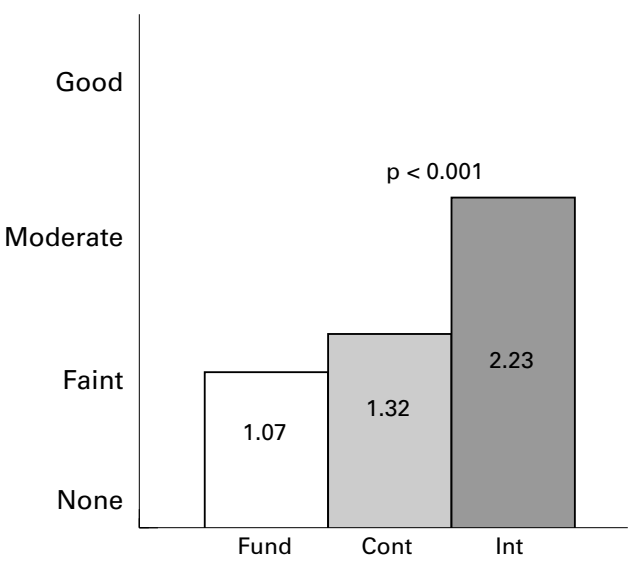

Figure 4 Qualitative analysis of peak contrast enhancement effect for all organs at the optimal perfusion volume using a visual scoring system. $0=$ none; 1 = faint; $2=$ moderate $; 3=$ good . Cont, continuous harmonic imaging; Fund, fundamental imaging; Int, intermittent harmonic imaging.

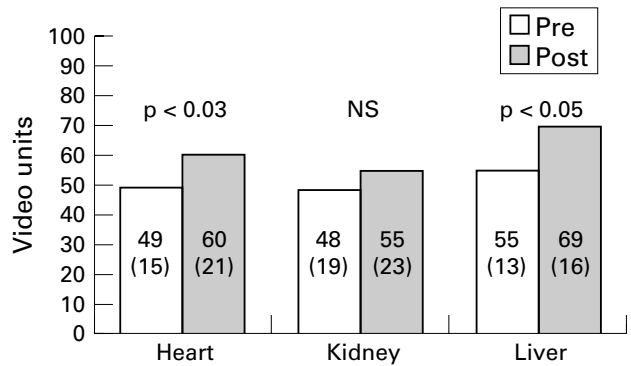

Figure 5 Peak video grey scale intensity at baseline and after contrast injection at the optimal perfusion volume for all organs for continuous harmonic imaging. Values are mean (SD).

INTEROBSERVER VARIABILITY

The two independent observers achieved agreement in 162 of 225 scores (72\%) when performing qualitative assessment of the contrast enhancement effect at the optimal perfusion volume. Agreement to within one score was achieved in 196 of 225 assessments (87\%). This represents good to excellent agreement.

QUANTITATIVE ANALYSIS

The results of the videodensitometric analysis to assess the change in peak harmonic grey scale from baseline following contrast injection for continuous harmonic imaging for all organs are shown in fig 5 . The mean change in peak harmonic grey scale was 11 video grey scale units (95\% confidence interval 2.4 to 19 ) for heart ( $\mathrm{p}<0.03), 7$ units $(-8$ to 22$)$ for kidney (NS), and 14 units (1.7 to 27 ) for liver $(\mathrm{p}<0.05)$.

The results for intermittent harmonic imaging (every second cardiac cycle) for all organs are shown in fig 6 . The change in peak harmonic grey scale level was 33 video grey scale units $(95 \%$ confidence interval 25 to 41.5) for heart ( $\mathrm{p}<0.0001), 24$ units (12 to 37) for kidney $(\mathrm{p}<0.0002)$, and 16 units $(6.5$ to 26.5) for liver $(p<0.001)$, showing a significant improvement in contrast effect comparing all intermittent modes with continuous imaging.

Comparing the contrast enhancement effect in each organ between the different patient

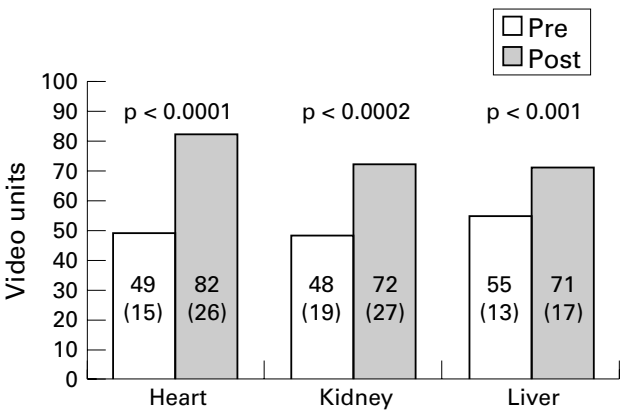

Figure 6 Peak video grey scale levels at baseline and following contrast injection at the optimal perfusion volume for all organs during intermittent harmonic imaging every second cardiac cycle. Values are mean (SD).

subgroups did not reveal any significant differences. The peak change in contrast enhancement effect in the heart was 14.6 for normal subjects, 16.9 for those with impaired left ventricular function and/or chronic pulmonary disease and normal hepatic and renal function, and 20.6 for those with left ventricular dysfunction, chronic pulmonary disease, hepatic impairment, and/or renal impairment (NS). For the kidney these figures were 14.2, 16 , and 24.9 (NS), and for the liver they were 13, 17.2, and 23.6, respectively (NS).

ADVERSE EVENTS

One subject who was diabetic with very poor left ventricular function had a rigor two hours after the study and developed a fever of $38.8^{\circ} \mathrm{C}$ and flu-like symptoms six hours after the study. Paracetamol was given to lower the temperature. All symptoms had resolved by the following day. There were no changes on the 12 lead ECG or biochemical profile. However the white cell count rose from $8.87 \times 10^{9} /$ litre before the study to $17.06 \times 10^{9} /$ litre 30 minutes after the study, with a neutrophilia of $91.7 \%$. Blood cultures were negative. This picture had resolved 24 hours after the study and may have reflected an adverse reaction to the contrast agent.

No other subjects had adverse reactions and there were no changes in their physical examination, 12 lead ECG, or biochemical and haematological profiles.

\section{Discussion}

The detection of myocardial (or other organ tissue) perfusion following intravenous contrast injection has previously been limited by the ability of the contrast agent to survive transpulmonary passage, as well as by the limitations of the ultrasound technology to detect the presence of contrast within the tissue. More recently, second generation contrast agents have been developed which can survive transpulmonary transit. Optison is such an agent, consisting of albumin microspheres filled with octafluoropropane. This is a high molecular weight gas which does not diffuse out of microbubbles as readily as air. This means that the microbubbles retain their shape and backscatter properties during transpulmonary transit, unlike first generation ultrasound contrast agents that contain air, which is highly 
diffusible and rapidly escapes from bubbles when mixed with blood; since the backscatter of a bubble is related exponentially to its radius, loss of air results in decreased bubble size which, in turn, leads to a decrease in its backscatter properties. Microbubble destruction following resonance would cause further loss of backscatter in a less stable contrast agent.

Contrast microbubbles resonate at transmitted ultrasound frequencies. They release ultrasound energy both at this frequency and at harmonics of this frequency. Unlike tissue, therefore, they have non-linear ultrasound backscatter properties. The development of ultrasound transducers that can transmit at one frequency and receive at the second harmonic of (that is, twice) that frequency has enhanced the ability to detect contrast microbubbles within the tissue. This is because, although the signal amplitude or backscattered energy is greater at the fundamental (transmitted) frequency, the difference between the tissue effect and the contrast effect is much greater at the second harmonic frequency since tissue generates less second harmonic signal than contrast. ${ }^{16}$

Many contrast microbubbles are destroyed by diagnostic ultrasound intensities. Therefore, reducing ultrasound intensity-either by reducing the transmit power or by reducing the time between insonations-allows accumulation of contrast agent and enhances the ability to detect its presence within the organ tissue of interest. ${ }^{4} 617$

Myocardial opacification following intravenously injected Optison has been studied with these ultrasound techniques in animal models. Studies in dogs during coronary occlusion have shown perfusion defects during occlusion which resolve following restoration of normal flow. ${ }^{8}$ Perfusion defects also correlate well with wall motion abnormalities and risk area determined by technetium scanning during coronary occlusion. ${ }^{18} \mathrm{~A}$ study comparing Optison with its parent compound Albunex showed that Optison was superior for evaluation of left ventricular chamber opacification and endocardial border delineation. ${ }^{19}$ Intermittent harmonic imaging during intravenous Optison injection in dogs shows good myocardial opacification. ${ }^{6}$ Perfusion defects occurred during adenosine induced hyperaemia in the presence of coronary stenoses during intermittent harmonic imaging which were difficult to measure during fundamental or continuous harmonic imaging. ${ }^{5}$ The magnitude of the perfusion defect using intermittent harmonic imaging correlated well with the magnitude of flow mismatch when radiolabelled microspheres were used, as well as with risk area and infarct size following coronary occlusion and reperfusion determined at necropsy. One study in humans has shown that myocardial contrast echocardiography following intravenous Optison can define the presence of coronary artery disease at rest and during pharmacological stress. The location of perfusion defects and their clinical relevance was comparable to the results of technetium sestamibi single photon emission computed tomography (SPECT). ${ }^{9}$

In the present study we have not examined the ability of Optison to demonstrate coronary artery disease, but rather its use in demonstrating myocardial, kidney, and liver perfusion at low doses in a variety of clinical conditions which may affect the transpulmonary passage, uptake, and persistence of the contrast agent in these organs. Our study has shown that Optison can opacify organ tissue at very low doses (as little as $0.1 \mathrm{ml}$ ) following intravenous injection. These doses are much lower than are necessary with the parent compound, Albinex. Previous studies with Optison in open chest dogs with the ultrasound transducer on the epicardial surface have used similar doses. The only other published study in human myocardium used doses of 0.5 or $1 \mathrm{ml}$. The optimal perfusion volume for each organ was less than $2 \mathrm{ml}$ during fundamental imaging. Intermittent second harmonic imaging, insonating at one frame every second cardiac cycle, provided the optimum imaging mode for all organs, increasing the peak change in video grey scale intensity from 11 to 33 units for myocardium $(\mathrm{p}<0.0001)$, from 7 to 24 units for kidney $(\mathrm{p}<0.0002)$, and from 14 to 17 units for liver $(\mathrm{p}<0.002)$ compared with continuous harmonic imaging. This was also appreciated visually by two independent observers, intermittent harmonic imaging achieving a score of 2.33 (moderate to good) compared with fundamental and continuous second harmonic imaging, which achieved scores of 1.07 and 1.32 respectively (faint). The presence of left ventricular function or chronic pulmonary disease with pulmonary hypertension did not seem to reduce the ability of the contrast agent to demonstrate organ perfusion, the peak change in video grey scale being similar to that in normal subjects (14.6 $v 16.9$ for myocardium (NS), $14.2 v 16$ for kidney (NS), and 13 $v 17.2$ for liver (NS)). There were no significant differences in kidney and liver enhancement compared with normal subjects in the presence of renal or hepatic dysfunction (14.2 $v 24.9$ for kidney and $13 v 23.6$ for liver). Rather, any difficulty in perceiving contrast enhancement seemed to depend entirely on the imaging mode (intermittent harmonic imaging being superior in all cases) and the quality of the baseline image.

\section{LIMITATIONS OF THE STUDY}

While this study involved only 25 patients, 505 intravenous injections of Optison were given, which did enable an analysis of the optimum perfusion volume for each organ as well as a comparison of the tissue contrast enhancement between the different imaging modes. Since harmonic imaging was so superior to fundamental imaging, an analysis of intermittent fundamental imaging was not performed. Although we made some comparisons between the patient subgroups with respect to the contrast enhancement effect (five normal subjects $v 10$ subjects with impaired left ventricular function and/or chronic pulmonary disease and normal renal and hepatic function, and 10 
subjects with abnormal renal and/or hepatic function), these groups are rather small. Certainly, more detailed statistical analysis of subgroups has not been possible.

\section{FUTURE DIRECTIONS}

Since intravenous Optison can demonstrate organ perfusion in patients with a variety of clinical conditions, particularly in conjunction with intermittent harmonic imaging, it may have many uses in the clinical setting. As has been shown, it may be useful in evaluating reversible ischaemia in patients with ischaemic heart disease. It may also be useful after myocardial infarction to determine noninvasively which patients have viable myocardium in the territory of their infarct related artery and so might benefit from invasive investigation. It should be possible to determine the patency of the infarct related artery, which would rationalise invasive management.

\section{CONCLUSIONS}

In this study we have shown the ability of an ultrasound contrast agent such as Optison to opacify myocardium, kidney, and liver tissue following low dose intravenous injection. This is possible even in patients with conditions which may affect the transpulmonary transit of the agent, the circulation of the agent to the tissues, and its ability to opacify the tissues. Intermittent second harmonic imaging enhances this effect. The increased availability of this imaging technology will enable second generation contrast agents such as Optison to become a useful non-invasive tool to evaluate organ perfusion in a variety of clinical conditions. This may be more cost effective and acceptable to patients than techniques currently available.

$\mathrm{HD}$ is a full time employee of Molecular Biosystems Inc.

1 Skyba DM, Camarano G, Goodman NC, et al. Hemodynamic characteristics, myocardial kinetics and microvascunar ich graphic contrast agent capable of producing myocardial opacification from a venous injection. F Am Coll Cardiol
2 Mulvagh SL, Foley DA, Aeschbacher BC, et al. Second harmonic imaging of an intravenously administered echocardiographic contrast agent. Visualisation of coronary arteries and measurement of coronary blood flow. $\mathcal{F ~} \mathrm{Am}$ Coll Cardiol 1996;27:1519-25.

3 Kaul S. New developments in ultrasound systems for contrast echocardiography. Clin Cardiol 1997;20(suppl 1):I27-30.

4 Villarraga HR, Foley DA, Aeschbacher BC, et al. Destruction of contrast microbubbles during ultrasound imaging at conventional power output. $\mathcal{F} \mathrm{Am}$ Soc Echocardiogr 1997;10:783-91.

5 Firschke C, Lindner JR, Wei K, et al. Myocardial perfusion imaging in the setting of coronary artery stenosis and acute myocardial infarction using venous injection of a second generation echocardiographic contrast agent. Circulation 1997;96:959-67.

6 Colon PJ, Richards DR, Moreno CA, et al. Benefits of reducing the cardiac cycle triggering frequency of ultrasound imaging to increase myocardial opacification with FS069 during fundamental and second harmonic imaging. 7 Am Soc Echocardiogr 1997;10:602-7.

7 Dittrich HC, Kuvelas T, Dadd K, et al. Safety and efficacy of the ultrasound contrast agent FS069 in normal humans: results of a phase I trial [abstract]. Circulation 1995; 92(suppl):I-464.

8 Meza MF, Greener Y, Aristizabal D, et al. Myocardial contrast echocardiography: safety of FS069, a new transpulmonary echocardiographic contrast agent. I $\mathrm{Am}$ Soc Echocardiogr 1994;7(suppl)

9 Kaul S, Senior R, Dittrich H, et al. Detection of coronary artery disease with myocardial contrast echocardiography.
Comparison with ${ }^{99 \mathrm{~m}} \mathrm{Tc}$-sestamibi single photon emission computed tomography. Circulation 1996;96:785-92.

10 Leen E, McArdle CS. Ultrasound contrast agents in liver imaging. Clin Radiol 1996;51(suppl 1):35-9.

11 Jakobsen J. Echo enhancing agents in the renal tract. Clin Radiol 1996;51 (suppl 1):40-3.

12 Albrecht T, Cosgrove DO, Correas JM, et al. Renal, hepatic and cardiac enhancement on Doppler and gray scale sonograms obtained with EchoGen. Acad Radiol 1996;3(suppl 2):S198-200

13 Kono Y, Moriyasu F, Nada T, et al. Gray scale second harmonic imaging of the liver: a preliminary animal study. Ultrasound Med Biol 1997;23:719-26.

14 Cosgrove D. Ultrasound enhancement of tumours. Clin Radiol 1996;51:44-9.

15 Forsberg F, Goldberg BB, Liu JB, et al. On the feasibility of real time, in vivo harmonic imaging with proteinaceous microspheres. F Ultrasound Med 1996;15:853-60.

16 Burns PN. Harmonic imaging with ultrasound contrast agents. Clin Radiol 1996;51:50-5.

17 Wei K, Skyba DM, Firschke C, et al. Interactions between microbubbles and ultrasound: in vitro and in vivo observations. F Am Coll Cardiol 1997;29:1081-8.

18 Camarano GP, Ismail S, Goodman C, et al. Assessment of risk area during coronary occlusion and infarct size after reperfusion can be determined with myocardial contrast echocardiography using intravenous injections of FS069, a new contrast agent. Circulation 1994;90(suppl):I-68.

19 Dittrich HC, Bales GL, Killam AL. Comparative study for the left ventricular opacification and endocardial border delineation following intravenous administration of Albunex and FS069 in the anaesthetised canine. (Summary report 1041.) San Diego: Molecular Biosystems Inc, 1994. 